\title{
Study of Geodetic datum of Nepal, China and Pakisthan and its transformation to World Geodetic System
}

\author{
Niraj Manandhar \\ Chief Survey Officer, Survey Department, Geodetic Survey Branch \\ manandhar_niraj@hotmail.com
}

\section{Abstract:}

There are many government, non-government and International organizations dealing in compilation of database or electronic maps with data from different sources often becomes necessary to transform from one coordinate system to another in order to work within a single unifying framework.

The significance of the study of the coordinate system of China, Pakistan, Nepal will benefit many researchers working in various disciplines and using geographic information system a part of their research. The use of remotely sensed data for environmental studies, disaster related matters and many more has widely been in use due to the availability of high resolution satellite images but it requires to have a relationship between the satellite images and ground location called geo-referencing. To establish this relationship the understating of the coordinate system is needed and also integrating to the national grid of the particular country if required then further knowledge of the parameters of datum chosen by the particular country will be a must. This paper illustrates some findings of the coordinate system of this region.

\section{Introduction:}

The national and regional surveying and mapping works are all based on one single framework of geodetic control which is considered as a primary network of the country. The importance of the geodetic frame work for the country is beyond justification and is applied in almost all development works of the country such as defense, satellite lunching, missiles projecting etc, construction of major infrastructures of the country (dams, roads, sewerage system, irrigation, hydropower stations etc).

The development of the Geographic Information System since last two decades has exemplified its importance and widened its application in many more sectors such as in the study of environmental change, disaster management, and creating the digital database in developing an information system.

The concept of the position defined by co-ordinate system is the most essential part in the process of map-making and to the performance of the spatial search and analysis of geographic information. In order to plot the geographical feature on the map it is necessary to define the position of points on the features with respect to a common frame of reference or the co-ordinate system in other words.

In the other hand all the observations and measurements for accurate mapping are carried out on the physical surface of the earth where as computations and representation of the earth surface into paper in the form of map requires mathematical figure of the earth. In other words there has to have some relationship between the mathematical earth (ellipsoid) and the physical earth (geoid). In the geodetic terms we need to define best fitting ellipsoid and geoid. The best fitting ellipsoid is that particular ellipsoid which best fits the spread of the earth surface of a particular country. Therefore there are different ellipsoids of different country and hence different countries have different origin and different datum defined by certain parameters.

The positions on the earth's surface are normally defined in two systems Cartesian and the Geographic co-ordinate system. In the Cartesian co-ordinate system positions are defined by their perpendicular distances from the set of fixed axes. Different values are specified for these axes in different countries.

The geographical coordinates constitute degrees of latitude and longitude. This is a form of spherical polar coordinate system in which two angles are measured with respect to 
the planes through the center of the ellipsoid representing the shape of the earth.

The latitude and longitude refer to the position in 3D space and for the cartographic works it is necessary to transform them in 2D map grid system. Such transformation is called projection. The projection refers to the transformation of the earth's surface either directly to a plane or to a cylindrical or a conical surface which having been conceptually warped around the earth which then form a flat surface when unrolled.

The choice of projection in a particular country is usually governed by a desire to minimize the distortion to best possible extent. Therefore it is important to appreciate the process of map projection and the way in which they introduce internal changes in scale and give rise to these distortions.

As a consequence due to the adoption of variety of map projection there are different map-grid co-ordinate systems in use among them some of which are unique to a particular mapping organization of the country.

\section{Aims and Objective of the study:}

Study the different datum position parameters adopted by different countries, Nepal, Pakistan and China in particular.

Find out the parameters used in the datum transformations between the adopted datum into internationally accepted World Geodetic System 1984 (WGS 84).

\section{Geodetic Datum:}

The primary or the first order network is defined by means of well defined three-dimensional reference system of co-ordinates related to the earth fixed reference system. Such a reference system is defined by the dimension of the reference ellipsoid in terms of five parameters such as semi-major axis ' $a$ ' and flattening ' $\mathrm{f}$ ' and its position represented by regional $\mathrm{X}, \mathrm{Y}, \mathrm{Z}$ or $\square, \lambda, \mathrm{h}$ system specifying the orientation with respect to the global $\mathrm{Xg}$, Yg, Zg system, hence with respect to earth or geoid.

Usually the centre of the ellipsoid does not coincide with the earth's centre of mass but that axes are made parallel to the earth's axis of rotation with a pre assumption that global Xg, Yg, Zg rectangular co-ordinate system, has the origin which lies on the earth's centre of mass and a $\mathrm{Z}$ axis coinciding with the mean rotating axis of the earth, $\mathrm{X}$ axis passing through the mean of the Greenwich meridian. The $\mathrm{Y}$ - axis as defined by the plane which is perpendicular to $\mathrm{X}$ and $\mathrm{Z}$ - axis (Torge, 1991, pl 38).
Determination of the parameters of such a reference datum defined by shape, size, and orientation of ellipsoid of revolution in other words the three dimensional coordinate system requires the high precision surface and spatial measurements. This work requires highly trained and skilled manpower.

\section{WGS-84 Co-ordinate System:}

The WGS-84 (World Geodetic System - 1984) is a Conventional Terrestrial System (CTS), realized by modifying the Navy Navigational Satellite System (NNSS), or TRANSIT, Doppler Reference Frame in origin and scale and rotating it to bring its reference meridian into coincidence with the Bureau International de l'Heure $(\mathrm{BIH})$ - defined Zero meridian.

The origin of WGS-84 system is the centre of mass of the earth. Its Z-axis lies along the direction of Conventional Terrestrial Pole (CTP) for polar motion and the X-axis lies along inter-section of the WGS-84 Reference meridian plane and the plane of CTP Equator. The Y-axis of this system completes a right-handed, Earth-Centered, EarthFixed (ECEF) orthogonal co-ordinates system, measured in the plane of CTP Equator $90^{\circ}$ degree East of X-axis.

The origin and orientation of co-ordinates axis in WGS-84 have been defined by the X,Y,Z co-ordinates established under the control of the 5 GPS monitoring stations located at Hawaii, Colorado Springs, Ascension, Diego Garcia and Kwajalein.

The WGS-84 is an earth-fixed global reference frame, including an earth model and is defined by a set of primary and secondary parameters. The primary parameters are as follows:

\begin{tabular}{|c|c|}
\hline Semi-major axis (a) & $6378137 \mathrm{~m}$ \\
\hline Flattening (f) & $1 / 298.257223563$ \\
\hline Angular velocity $(\omega)$ & $\begin{array}{l}7.292115 \mathrm{X} \quad 10^{(-5)} \text { (Radi } \\
\text { per second) }\end{array}$ \\
\hline \multicolumn{2}{|c|}{ Geocentric gravitational } \\
\hline Constant (GM) & $398600.5 \mathrm{Km}^{3} \mathrm{~s}^{-2}$ \\
\hline \multicolumn{2}{|c|}{ (Mass of the earth's atmosphere included) $\quad-484.16685 \times 10^{-6}$} \\
\hline \multicolumn{2}{|l|}{ Normalized $2^{\text {nd }}$ degree } \\
\hline \multicolumn{2}{|c|}{ zonal harmonic coefficient } \\
\hline of the gravitational c & \\
\hline
\end{tabular}

\section{Nepal Datum:}

Geodetic Survey Branch (GSB) was aware of the requirement of National Geodetic datum defined by the network of points of first order controls. With the 
agreement between the government of Nepal and the United Kingdom's Directorate of Military Survey, Ministry of Defense (MODUK) established the first order geodetic control net in the country. The task was completed in 1986.

\section{The Datum was defined as in above:}

References ellipsoid: $\quad$ Everest (1830)

Semi-major axis (a) $6377276.345 \mathrm{~m}$

Flattening (f) $\quad 1 / 300.8017$ and $\left(\mathrm{e}^{2}=0.00663784663\right)$

With the Geodetic Origin Station 12/57 Nagarkot defined as

$$
\begin{aligned}
& \text { Latitude }(\square \mathrm{g})=27^{\circ} 41^{\prime} 31^{\prime} .04 \mathrm{~N} \\
& \text { Longitude }(\lambda \mathrm{g})=85^{\circ} 31^{\prime} 20^{\prime} .23 \mathrm{E} \\
& \text { meridian }(\xi)=-37^{\prime} .03 \\
& \text { Prime Vertical }=-21^{\prime \prime} .57
\end{aligned}
$$

and assuming the geoid height

$$
(\mathrm{N})=0 \text { meter }
$$

The deflections quoted are derived from an astronomic position observed by Czechoslovak Geodetic Institute.

The Nepal datum represents a rigorous reference system. The net in properly oriented to the conventional origin (CIO) and the scale of the net is consistent with the international standards of length defined by the Doppler satellite observation.

As stated in the Report submitted by MODUK, the geographical co-ordinates of first order points are of high standard and hence fulfill the requirement of a rigorous Geodetic datum in Nepal.

\section{Pakistan Datum:}

The Everest Ellipsoid has been in use in several countries of Indian Sub-continent for the mapping activities. Named after Sir George Everest the ellipsoid was derived in 1830 and since then it has been used as a basis for all types of control surveys. Sir George Everest paid careful attention to the measurement of bases and astronomical latitude and longitude were measured through out the arc of the meridian especially at Kalianpur in Madhya Pradesh.

Dimensions of the Everest ellipsoid and its orientation at origin were carried out one by one at a number of times. Semi-major axis (a), flattening (f) and northsouth component of deflection of vertical (Meridonal) was defined by Everest in 1840 where as East-West (prime vertical) component was defined by Walker in 1878. Though Everest ellipsoid has been the best fitting mathematical surface for India and adjacent countries but it cannot be extended too far from the origin and hence it application are rather limited. Therefore this ellipsoid has been marginally modified by the different countries of the Indian sub-continent.

The ellipsoidal parameter adopted by Pakistan are slightly different from Everest ellipsoid 1830.

The ellipsoidal parameters adopted by Pakistan are as follows:

$$
\begin{array}{ll}
\text { Ellipsoid : } & \text { Everest Pakistan } \\
\text { Semi-major axis (a): } & 6377309.613 \mathrm{~m} \\
\text { Flattening (f) : } & 1 / 300.8017 \quad \text { (source: NIMA } \\
& 8350.24^{\text {th }} \text { July 1977) }
\end{array}
$$

The values of the datum origin of Pakistan were not found during the literature review. The great triangulation chain of India was extended to Pakistan at the time of Sir Geogre Everest. therefore possibly Kalianpur could be the datum origin.

\section{Chinese Datum:}

During the literature review it was found that Central Bureau of Land Survey of China

( CBLS) established the Nanking Datum of 1935 where:

$$
\begin{aligned}
& \Phi_{0}=32^{0} 04^{\prime} 19.7445^{\prime} \text { North, } \\
& \Lambda_{0}=118^{0} 50^{\prime} 18.5354^{\prime} \text { East of Greenwich. }
\end{aligned}
$$

The ellipsoid of reference is the International (also called the Hayford 1909 and the Madrid 1924) where:

$$
\begin{array}{ll}
\text { Semi-major axis (a) } & 6,378,388 \text { meters, and } \\
\text { Flattening } 1 / \mathrm{f} & 297
\end{array}
$$

A Gauss-Kruger Transverse Mercator Grid is defined at the Datum origin. The scale factor at origin $\left(\mathrm{m}_{0}=1.0\right)$; the False Easting and the False Northing = zero.

It is also found that the Current Grid Systems attributed to the People's Republic of China find their roots in the Russian (USSR) origins of assistance. For instance, the Russia Belts for China are identical with the UTM specification with the exceptions or variations that the scale factor at origin is unity rather than 0.9996 . 
The ellipsoid of reference is the Krassovsky 1940 where:

Semi major axis (a) 6,378,245 m, and

Flattening (1/f) 298.3.

A variation on this is known as the three degrees Belts, and the location of the Central Meridians are simply a (half) scalar of the six degrees belts.

\section{Datum Transformation parameters:}

WGS-84 coordinate system is being adopted universally as the standard form of Geographical Coordinate Representation System. World wide development in the GPS and GIS system and its international adoption has created an environment in developing a common base of reference in exchanging the geographic data. This system came into existence only towards the end of $20^{\text {th }}$ Century. Prior to that local coordinate system were in use. Thus most of the maps, records and data are available in local systems of the particular country. The coordinates, maps and records related to land are considered of very important property of the nation from the defense point of view therefore these information are kept secret by the nation and its national policy. The precise transformations parameters of the country are not made available to users still today.

In India, Pakistan and China still the topographical maps based on particular datum of the country is not freely available to the users. They are kept secret from the defense point of view. In this context this study has limitations to acquire such information.

With the increasing exchange of geographic information locally and globally positions defining the location need to be available in terms of both locally adopted datum and global datum. The process of mathematical conversion of the positions from one system to another is called datum transformation.

\section{Transformation models:}

Several mathematical models have been developed which describe the functional relationship between pairs of three dimensional coordinates. The two most commonly used mathematical models to transform positions between the reference systems are

Bursa-Wolf (Bursa, 1962, Wolf, 1963) and

Molodensky (Molodensky et.al., 1962)

These are the standard models due to their extensive use around the world over a number of years. The only difference between these two methods is Molodensky uses local origin about which the transformation is performed where as Bursa-Wolf method uses reference system origin. It has been shown that both methods gives the identical results provided full statistical information (variance and covariance of parameters and positions) is carried out through the transformation process (Harvey, 1996). In most cases Bursa-Wolf method is preferred as it does not require local origin coordinates to be maintained along with the transformation parameter equation.

\section{BURSA-Wolf transformation model:}

The Bursa-Wolf method assumes a similarity three dimensioned relationship between two consistent sets of Cartesian coordinate through seven parameters:

- three translations $(\Delta \mathrm{X}, \Delta \mathrm{Y}, \Delta \mathrm{Z})$

- three rotations around $\mathrm{X}, \mathrm{Y}, \mathrm{Z}$ axis respectively $(\varepsilon, \psi, \omega)$

- a scale change $(\Delta \mathrm{L})$

If $\mathrm{U}, \mathrm{V}$ and $\mathrm{W}$ represent the Cartesian components of a station in reference frame 1 say Everest and $\mathrm{X}, \mathrm{Y}, \mathrm{Z}$ represent the Cartesian component of same stations in reference frame number 2 say WGS-84, the transformation can be expressed as:

$$
\left|\begin{array}{l}
X \\
Y \\
Z
\end{array}\right|=\left|\begin{array}{l}
\Delta X \\
\Delta Y \\
\Delta Z
\end{array}\right|+(1+\Delta L) R\left|\begin{array}{l}
U \\
V \\
W
\end{array}\right|
$$

where $\mathrm{R}$ represents a $3 \times 3$ rotation matrix and defined a

$$
\mathrm{R}=\mathrm{R}_{1}(\varepsilon) \mathrm{R}_{2}(\psi) \mathrm{R}_{3}(\omega)
$$

If all three angles are small the above rotation matrix can be written in its simplified form by setting sine of an angle equal to the angle itself, cosine of the angle equal to 1 and the product of sines equal to zero.

Thus after simplification the above matrix will appear as

$$
\mathrm{R}=\left|\begin{array}{lll}
1 & \omega & -\Psi \\
-\omega & 1 & \varepsilon \\
\psi & -\varepsilon & 1
\end{array}\right|
$$


The transformation equation (4-1) can now be written as

$$
\left|\begin{array}{l}
X \\
Y \\
Z
\end{array}\right|=\left|\begin{array}{l}
\Delta X \\
\Delta Y \\
\Delta Z
\end{array}\right|+(1+\Delta L)\left|\begin{array}{lll}
1 & \omega & -\psi \\
-\omega & 1 & \varepsilon \\
\psi & -\varepsilon & 1
\end{array}\right|\left|\begin{array}{l}
U \\
V \\
W
\end{array}\right|
$$

\section{Method of estimation of transformation parameters}

A point physically identifiable on the surface of Earth which has been assigned coordinates in at least two separate systems of coordinates is termed as collocated station. The Cartesian coordinates of sufficient number of collocated stations (U, V, W, X, , Z) can be used as observations in a least square adjustment for the seven transformation parameters. The model in symbolical form can be written as:

$$
\mathrm{F}(\mathrm{L}, \mathrm{X})=0
$$

where

$$
\begin{aligned}
& \mathrm{L}=\text { observations }(\mathrm{U}, \mathrm{V}, \mathrm{W}, \mathrm{X}, \mathrm{Y}, \mathrm{Z}) . \\
& \mathrm{X}=\text { parameters }(\Delta \mathrm{X}, \Delta \mathrm{Y}, \Delta \mathrm{Z}, \Delta \mathrm{L}, \omega, \psi, \varepsilon)
\end{aligned}
$$

By arranging the equations (3) into this form will result in

$$
\begin{aligned}
& \Delta \mathrm{X}+\mathrm{U}+\mathrm{w} \mathrm{V}-\mathrm{y} \mathrm{W}+\Delta \mathrm{L} \mathrm{U}+\mathrm{w} \Delta \mathrm{LV}-\psi \Delta \mathrm{LW}-\mathrm{X}=0 \\
& \Delta \mathrm{Y}+\mathrm{V}-\mathrm{w} \mathrm{U}+\mathrm{e} \mathrm{W}+\Delta \mathrm{LV}-\mathrm{w} \Delta \mathrm{L} \mathrm{U}+\mathrm{e} \Delta \mathrm{L} \mathrm{W}-\mathrm{Y}=0 \\
& \Delta \mathrm{Z}+\mathrm{W}+\psi \mathrm{U}-\varepsilon \mathrm{V}+\Delta \mathrm{L} \mathrm{W}+\psi \Delta \mathrm{L} \mathrm{W}-\varepsilon \Delta \mathrm{LV}-\mathrm{Z}=0
\end{aligned}
$$

These three equations represent the functional relationship between any two closely oriented, closely scaled, ortho normal Cartesian coordinates systems. Since the observations (6 Cartesian components per station) have systematic and other errors with them, the usual combined least squares procedure of minimizing the weighted sum of residuals squared ( $\left.\mathrm{V}^{\mathrm{T}} \mathrm{PV}\right)$ is followed. in order to transform the digital data of the topographical maps or the ground control points (GCP).

The topographical maps of Nepal are prepared and published in two parts. One of the Eastern Nepal and other of the Western Nepal. In both cases the ground controls for the topographical map preparation were established by Global positioning system (GPS) technique. Since the maps thus prepared have to be based on Everest Ellipsoid 1830, Nepal Datum the GPS established ground control points has to be transformed from WGS-84 to Everest Ellipsoid 1830.

In the process of the determination of the transformation parameter (the common points between the reference frame i.e. WGS-84 and Everest 1830) first order points based on Nepal datum, Everest 1830 only 11 points were used as the common points.

The values of the transformation (WGS-84 to Local) parameters are as follows:

$\Delta X=-293.17 m, \Delta Y=-726.18 m, \Delta Z=-245.36 m$

Using these parameter values, all WGS-84 co-ordinates were transformed to Nepal datum, Everest 1830 (Geoid Studies for Nepal, 1997)

Since Nepal datum represents a rigorous reference system and net in properly oriented to the conventional origin (CIO) and the scale of the net is consistent with the international standards of length defined by the Doppler satellite observation the rotation between the two reference frame ie WGS-84 and Nepal datum, Everest 1830 is considered as zero and 1-sigma accuracy of the determination of transformation parameter is 0.26 $\mathrm{m}$. The test results shows that it can be applied for the transformation of the topographical database of Nepal. This test is independently carried by the author for his research work and the users are advised to use it in their own risk.

\section{Datum Transformation Parameter of Pakistan:}

Datum transformation Parameter from (WGS-84 to Local) can be considered as follows:

$\Delta X=-283 m, \Delta Y=-682 m, \Delta Z=-231 m$

(source: NIMA $8350.24^{\text {th }}$ July 1977)

This transformation parameters tend to be of regional nature. This result may not be sufficiently accurate
The present study is focused on the estimation of transformation parameters between Everest and WGS-84 
for transforming the topographic database and in the transformation of geodetic ground control points although its accuracy is specified $+/-1 \mathrm{~m}$ in $\mathrm{X}, \mathrm{Y}$ and $\mathrm{Z}$ coordinates.

More accurate transformation parameters can be acquired by further communication with National Mapping Organization of Pakistan.

\section{Datum transformation parameter of China:}

During the author's visit to National Geomatics Center of China, Beijing; with the personal communication with the officials of the Geomatics Center new coordinates and elevations of the geodetic controls are based on internationally applied WGS-84, ITRF 2000 ( International Terrestrial Reference Frame 2000 ), the geoid model derived from EGM 96 ( Earth Gravity Model 96 ) Elevation Datum, and the Gauss-Krueger projection by 6 degree zone.

The most of the topograchical data base of China is now available in WGS-84 system however the transformation parameters between two system is not made available to the users. Therefore due to the availability of the data in WGS-84 the methods of conversion can be skipped while using the data base based on WGS-84.

\section{Discussion:}

In order to work with data from different sources all data must be transformed into one standard coordinate system. This can be either geographical coordinate or the rectangular co-ordinates system of the base data. Since for the mapping purposes Cartesian co-ordinates becomes necessary. Therefore the best approach would be to transform all data into WGS-84 system. Then the data can be superimposed with each other using different GIS related software.

Therefore Cartesian coordinates shoud be converted to Geographical coordinates i.e Latitude, Longitude and Height. Then by using the appropriate transformation parameters, it is converted into Geographical coordinates in WGS-84 system and these coordinates is then further transformed into rectangular coordinates in WGS-84 in case of the topographical data base of Nepal.

Similarly Cartesian co-ordinates based on Local Coordinate System (LCC) is transformed into Geographical coordinates. Then the transformed geographical coordinates is converted into WGS-84 geographical. It is further transformed into the rectangular coordinates in WGS-84. So that both data bases of two different countries based on different projections can be brought into a common platform.

\section{References:}

1. Geodesy, G. Bomford $3^{\text {rd }}$ Edition.

2. Military Engineering Vol. XIII- Part VI, Survey Computations 1978.

3. Manandhar, N. (1997) Study Report on Geodetic Datums, Transformation between the Reference System and Height Datum in Nepal, Survey Department, Geodetic Survey Branch (Unpublished).

4. Manandhar, N. (1997) The Geoid Studies in Nepal, M.Sc thesis, University of New South Wales, Australia.

5. R.R. Chhatkuli, S.M. Shrestha, N. Manandhar (2003), Transformation of Datum and Projection of Spatial data in GIS Application in the context of NGII in Nepal. National Convention and FEISCA Regional Meet.

6. Report of Ground Control Survey, Eastern Nepal Topographical Mapping Project, Survey Department.

7. Final Report of Ground Control Surveys, Vol.I, Vol. II, Vol.III Western Nepal Topographical Mapping Project, Survey Department. 